\title{
Overexpression of the medium-conductance calcium-activated potassium channel (SK4) and the HCN2 channel to generate a biological pacemaker
}

\author{
HONGYI ZHAO ${ }^{1-3^{*}}$, MEI YANG ${ }^{1-3^{*}}$, FENGYUAN WANG $^{1-3}$, ANKANG YANG $^{1-3}$, QINGYAN ZHAO $^{1-3}$, \\ XI WANG $^{1-3}$, YANHONG TANG ${ }^{1-3}$, TENG WANG ${ }^{1-3}$ and CONGXIN HUANG ${ }^{1-3}$ \\ ${ }^{1}$ Department of Cardiology, Renmin Hospital of Wuhan University; ${ }^{2}$ Cardiovascular Research Institute, \\ Wuhan University; ${ }^{3}$ Hubei Key Laboratory of Cardiology, Wuhan, Hubei 430060, P.R. China
}

Received January 18, 2019; Accepted July 25, 2019

DOI: $10.3892 / \mathrm{mmr} .2019 .10591$

\begin{abstract}
Ion channels serve important roles in the excitation-contraction coupling of cardiac myocytes. Previous studies have shown that the overexpression or activation of intermediate-conductance calcium-activated potassium channel (SK4, encoded by KCNN4) in embryonic stem cell-derived cardiomyocytes can significantly increase their automaticity. The mechanism underlying this effect is hypothesized to be associated with the activation of hyperpolarization-activated cyclic nucleotide-gated channel 2 (HCN2). The aim of the present study was to explore whether a biological pacemaker could be constructed by overexpressing SK4 alone or in combination with $\mathrm{HCN} 2$ in a rat model. Ad-green fluorescent protein (GFP), Ad-KCNN4 and Ad-HCN2 recombinant adenoviruses were injected into the left ventricle of Sprague-Dawley rat hearts. The rats were divided into a GFP group $(\mathrm{n}=10)$, an SK4 group $(\mathrm{n}=10)$, a HCN2 group $(n=10)$ and an SK4 + HCN2 (SK4/HCN2) group $(n=10)$. The isolated hearts were perfused at 5-7 days following injection, and a complete heart block model was established. Compared with the GFP group, overexpressing SK4 alone did not significantly increase the heart rate after establishment of a complete heart block model [98.1 \pm 8.9 vs. $96.7 \pm 7.6$ beats per min (BPM)], The heart rates in the SK4/HCN2 (139.9 $\pm 21.9 \mathrm{BPM})$ and $\mathrm{HCN} 2$ groups $(111.7 \pm 5.5 \mathrm{BPM})$ were significantly increased compared with the GFP and SK4 groups, and the heart rates in the SK4/HCN2 group were
\end{abstract}

Correspondence to: Professor Congxin Huang, Department of Cardiology, Renmin Hospital of Wuhan University, 238 Jiefang Road, Wuchang, Wuhan, Hubei 430060, P.R. China

E-mail: huangcongxing@vip.163.com

*Contributed equally

Key words: intermediate-conductance calcium-activated potassium channel, hyperpolarization-activated cyclic nucleotide-gated channel, heart rate, pacemaker significantly increased compared with the SK4 or HCN2 groups. In the HCN2 $(n=8)$ and the SK4/HCN2 $(n=7)$ groups, the shape of the spontaneous ventricular rhythm was the same as the pacing-induced ectopic rhythm in the transgenically altered site. By contrast, these rhythms were different in the SK4 $(n=10)$ and GFP $(n=10)$ groups. There were no significant differences in action potential duration alternans or ventricular arrhythmia inducibility between the four groups (all $\mathrm{P}>0.05$ ). Western blotting, reverse transcription-quantitative PCR and immunohistochemistry analyses showed that the expression levels of SK4 and HCN2 were significantly increased at the transgene site. Biological pacemaker activity could be successfully generated by co-overexpression of SK4 and $\mathrm{HCN} 2$ without increasing the risk of ventricular arrhythmias. The overexpression of SK4 alone is insufficient to generate biological pacemaker activity. The present study provided evidence that SK4 and HCN2 combined could construct an ectopic pacemaker, laying the groundwork for the development of improved biological pacing mechanisms in the future.

\section{Introduction}

Calcium-activated potassium channels can be categorized as large-conductance calcium-activated potassium channels, intermediate-conductance calcium-activated potassium channels (IK or SK4, encoded by KCNN4), or small-conductance calcium-activated potassium channels (SK1-3) according to their level of conductance (1).

SK4 is a non-voltage-dependent channel, and intracellular free calcium concentrations are sufficiently high to activate these channels. The channel is widely distributed in red blood cells, lymphocytes, monocytes, macrophages, epithelial cells and vascular smooth muscle cells (2). In addition, SK4 transcripts are found in embryonic stem cell-derived cardiomyocytes (ESC-CM), the mouse sinoatrial node, the adult human right atrium and in ventricular biopsies (3-6). Previous studies have shown that the overexpression or activation of SK4 in ESC-CM can significantly increase the duration of action potentials, elevate the trigger frequency and increase the beating area of cardiac embryoid bodies; these effects disappear after SK4 channel blockage, indicating that SK4 serves 
an important role in regulation of the cardiac rhythm $(3,4)$. A recent study has shown that SK4 is closely related to the pace function of the sinoatrial node in vivo (7). It was shown that the trigger frequency of the sinoatrial node decreased significantly following application of a specific inhibitor of SK4, and a mathematical model predicted that the trigger frequency of the sinoatrial node cells could be increased by the increasing SK4 current (6). A previous study postulated that the SK4 outward potassium current, which is responsible for the notch of the maximum diastolic potential (MDP), provides a driving force that is sufficiently strong to activate funny current [I(f) current] at the early phase of the diastolic depolarization (DD) slope (2).

Hyperpolarization-activated cyclic nucleotide-gated channel (HCN)2 is a member of the HCN channel family and its activity is affected by cAMP. Compared with HCN1, HCN2 and $\mathrm{HCN} 4$ both respond strongly to camp, but HCN2 has faster kinetics than HCN4, thus HCN2 was chosen for the present study. Previous studies have shown that the overexpression of HCN2 alone $(8,9)$ or in combination with other genes (10-13) can increase the beating frequency of cardiomyocytes, and promote in vivo pacemaker activity in a canine model of complete heart block. The aim of the present study was to explore whether the SK4 overexpression alone or combined with HCN2 could generate biological pacemaker activity. This was investigated in a rat model of complete heart block.

\section{Materials and methods}

Animals. The present study was approved by the Animal Studies Subcommittee of Wuhan University School of Medicine and was conducted in accordance with the Guide for the Care and Use of Laboratory Animals of the National Institutes of Health (14). All of the surgeries were performed under sodium pentobarbital anesthesia $(40 \mathrm{mg} / \mathrm{kg})$. After a $30-\mathrm{min}$ procedure, the animals were routinely treated with penicillin for 3 days to prevent infection, and then housed in a barrier environment until the end of the experiment. Prior to the heart extraction, the rats were anesthetized intraperitoneally with pentobarbital sodium (40 mg/kg). When deep anesthesia was established [respiratory depression, severe muscle relaxation, bradycardia, no reflexes (palpebral, corneal) and pupil dilation], the heart was removed for further experiments.

Adenovirus construction and purification. pHBAd-MCMV (Hanbio Biotechnology Co., Ltd.) was double digested with BamHI/AgeI. The ORF of the mouse KCNN4 gene (GenScript) was amplified via PCR using Taq polymerase (SinoBio Biotech). The sequences were: Kcnn4, forward 5'-GTTCTGCACGCT GAGATGTTG-3' and reverse 5'CTTGGCATGGAAGACCAC AAT-3'. The thermocycling conditions were pre-denaturation at $98^{\circ} \mathrm{C}$ for $5 \mathrm{~min}$, denaturation at $98^{\circ} \mathrm{C}$ for $10 \mathrm{sec}$, annealing at $55^{\circ} \mathrm{C}$ for $10 \mathrm{sec}$, and a final extension at $72^{\circ} \mathrm{C}$ for $90 \mathrm{sec}$, a total of 30 cycles. After enzyme digestion, gel extraction was performed. The digested fragment and vector were ligated to form pHBAd-MCMV-KCNN4, which was then transformed into competent DH5 $\alpha$ cells (Tiangen Biotech Co., Ltd.). Positive clones were identified by liquid sequencing. Large-scale preparation of recombinant plasmid was conducted using the Plasmid Midi Preparation kit (Beijing CW Biotech Co., Ltd.). 293 cells (from our laboratory; $60 \%$ confluence) were transfected with pHBAd-MCMV-KCNN4 $(1 \mu \mathrm{g} / \mu \mathrm{l})$ and the backbone vector pHBAd-BHG (Hanbio Biotechnology Co., Ltd.) using Lipofectamine $2000^{\circledR}$ (Thermo Fisher Scientific, Inc.). The supernatant was harvested after virus amplification (10 days). pHBAd-MCMV vector was also used for mouse HCN2 (M-HCN2, forward 5'-GTTCTGCACGCTGAGATGTTG-3' and reverse 5'-CTTGGCATGGAAGACCACAAT-3') and GFP construction. HCN2 vectors were prepared as described previously (15). The titers of Ad-GFP, Ad-HCN2 and Ad-KCNN4 were measured as $1 \times 10^{10} \mathrm{PFU} / \mathrm{ml}$ and preserved at $-80^{\circ} \mathrm{C}$.

Experimental protocol. A total of 40 male 8-week Sprague Dawley rats each weighing 220-250 g were purchased from Hunan Slac Jingda Laboratory Animal Co., Ltd. and raised in an SPF laboratory animal room at $20-26^{\circ} \mathrm{C}$ with humidity of $40-70 \%$ and fed with irradiated feed and sterile water. The rats were randomly divided into 4 groups: A GFP group $(n=10)$, a SK4 group $(n=10)$, a HCN2 group $(n=10)$ and a SK4/HCN2 group $(n=10)$. All of the surgeries were performed under sodium pentobarbital anesthesia $(40 \mathrm{mg} / \mathrm{kg})$. Once anesthetized, the rats were intubated, and a thoracotomy was performed on the left side to expose the heart. The free left ventricle wall was used as the transgene injection site. The injection site was marked by a line on the surface of the left ventricle, and the virus was injected with a microsyringe $\left(1 \times 10^{9} \mathrm{PFU} / \mathrm{ml}, 100 \mu \mathrm{l}\right)$.

Ex vivo electrocardiogram recording. At 5-7 days following cardiac injection (the levels of gene expression were highest during this time window; data not shown), when complete anesthesia was established in rats, a thoracotomy was conducted, and the hearts were quickly isolated, inserted into a Langendorff perfusion system (ADInstruments Ltd.) and secured for retrograde perfusion at $37^{\circ} \mathrm{C}$ with oxygenated Tyrode's solution at a rate of $6-8 \mathrm{ml} / \mathrm{min}$. The perfused heart was placed on a Sylgard-coated plate filled with warm Tyrode's solution (mM: $\mathrm{NaCl} 130, \mathrm{KCL} 5.4, \mathrm{CaCl}_{2} 1.8, \mathrm{MgCl}_{2}$ $1, \mathrm{Na}_{2} \mathrm{HPO}_{4}$ 0.3, HEPES 10 and Glucose 10; $\mathrm{pH}=7.4$, adjusted with $\mathrm{NaOH}$ ). ECG leads I and II were placed at appropriate sites. After a 20-min equilibration period, the atrioventricular node region was ablated with $75 \%$ alcohol using a microsyringe. After a complete heart block was stably established, the heart rate was recorded for a period of $20 \mathrm{~min}$ and electrode pacing was performed at the site of the transgene injection at 200-msec intervals.

Action potential duration (APD) alternans. Paired platinumstimulating electrodes were positioned on the basal surface of the right ventricle. The S1-S1 pacing protocol was performed with a series of pulse trains at a regular pacing cycle length (PCL). Starting at $150 \mathrm{msec}$, the PCL was shortened to $70 \mathrm{msec}$ in $10-\mathrm{msec}$ intervals. The regular pacing at each PCL lasted for $15 \mathrm{sec}$ to ensure a steady rhythm, and each pacing was separated by at least $30 \mathrm{sec}$ to minimize the pacing memory. The APD at a PCL of $300 \mathrm{msec}$ was determined at $90 \%$ repolarization (APD90) at each site. The APD alternans was assessed at each site by subtracting the APD90 for 2 consecutive beats when the alternate APD90 differed by 5\% over 10 beats. The threshold was defined as the maximal PCL (PCLmax) that induced APD alternans. 
Table I. Polymerase chain reaction primers used in this study.

\begin{tabular}{|c|c|c|}
\hline Gene & Primer sequences & Product size (bp) \\
\hline \multicolumn{3}{|l|}{ R-GAPDH } \\
\hline Forward & 5'-CGCTAACATCAAATGGGGTG-3' & 201 \\
\hline Reverse & 5'-TTGCTGACAATCTTGAGGGAG-3' & \\
\hline \multicolumn{3}{|l|}{ M-HCN2 } \\
\hline Forward & 5'-TCCTCATAGTGGAGAAGGGAATC-3' & 191 \\
\hline Reverse & 5'-ACAGATGCGCATCACTGCAC-3' & \\
\hline \multicolumn{3}{|l|}{ M-SK4 } \\
\hline Forward & 5'-GTTCTGCACGCTGAGATGTTG-3' & 126 \\
\hline Reverse & 5'-CTTGGCATGGAAGACCACAAT-3' & \\
\hline
\end{tabular}

Ventricular arrhythmia (VA) inducibility. Burst pacing protocols were performed to determine the VA susceptibility. Burst pacing (2-msec pulses at $50 \mathrm{~Hz}$ and two spacing durations) was performed at the left anterior free-wall, and repeated 3 times after a 2 -sec rest period. VA was defined as a run of $>2$ sec of consecutive premature ventricular contractions (wide QRS complexes). VA was considered non-sustained when the contractions lasted 2-30 sec and sustained when they lasted $>30 \mathrm{sec}$, according to established criteria (16). The VA vulnerability was evaluated based on the incidence of VA and the ratio of sustained VA.

Western blot analysis. Tissue specimens were obtained from the myocardium from the injection site and temporarily stored at $-80^{\circ} \mathrm{C}$ until assay. The tissue protein was extracted by RIPA Lysis Buffer (Aspen Technology, Inc.). Determination of protein concentration was the BCA method. Then, $5 \%$ of concentration gel and $10 \%$ of separation gel were chosen and the protein samples ( $40 \mu \mathrm{g}$ protein/lane) were mixed with $5 \mathrm{X}$ SDS-PAGE buffer (Aspen Technology, Inc.) in a water bath at $95-100^{\circ} \mathrm{C}$ for $5 \mathrm{~min}$, prior to being transferred to a polyvinylidene difluoride membrane. The expression of SK4 and HCN2 was measured using western blotting ( $n=10 /$ group). Membranes were incubated with the primary antibodies against GAPDH (loading control; 1:500; ab181602, Abcam), SK4 (1:500; ab215990, Abcam) and HCN2 (1:1,000; ab19346, Abcam). The membranes were blocked with $5 \%$ non-fat dry milk in tris-buffered saline with $0.05 \%$ Tween 20 (TBST) for $1 \mathrm{~h}$ at room temperature and incubated with the primary antibodies overnight at $4^{\circ} \mathrm{C}$. The membranes were then washed in TBST three times, incubated with horseradish peroxidase-conjugated anti-rat $(1: 10,000 ; 14-16-06)$ and anti-rabbit (1:10,000; 074-1506) secondary antibodies (SeraCare Life Sciences, Inc.) for $1 \mathrm{~h}$ at $37^{\circ} \mathrm{C}$, and imaged using Immun-Star (Bio-Rad Laboratories, Inc.) horseradish peroxidase substrate. The relative expression of the protein levels were determined using image analyzer software (AlphaEaseFC V; Alpha Innotech; ProteinSimple).

Reverse transcription-quantitative PCR (RT-qPCR). Total RNA was extracted from the myocardium $(100 \mathrm{mg})$ at the end of the study using TRIzol reagent $(1 \mathrm{ml})$ (Invitrogen; Thermo Fisher Scientific, Inc.). RT-qPCR was performed to evaluate the mRNA expression of KCNN4 and HCN2 (n=10/group). Isolated RNA $(2 \mu \mathrm{g})$ was converted into cDNA using a First Strand cDNA Synthesis kit (Toyobo Life Science) in a $15 \mu \mathrm{l}$ mixture as follows: $42^{\circ} \mathrm{C}$ for $2 \mathrm{~min}, 37^{\circ} \mathrm{C}$ for $15 \mathrm{~min}, 85^{\circ} \mathrm{C}$ for $5 \mathrm{~min}$ and $4^{\circ} \mathrm{C}$ for $10 \mathrm{~min}$. The primers (Table I) used for qPCR amplification were synthesized by Invitrogen (Thermo Fisher Scientific, Inc.). qPCR was performed using a StepOne Real-Time PCR system (Life Technologies, Thermo Fisher Scientific, Inc.) and SYBR® Premix Ex Taq ${ }^{\mathrm{TM}}$ II (Takara Bio, Inc.) as follows: Pre-denaturation at $95^{\circ} \mathrm{C}$ for $5 \mathrm{~min}$, denaturation at $95^{\circ} \mathrm{C}$ for $30 \mathrm{sec}$, annealing at $58^{\circ} \mathrm{C}$ for $20 \mathrm{sec}$, and a final extension at $72^{\circ} \mathrm{C}$ for $45 \mathrm{sec}$, a total of 40 cycles. The dissolution curve was from $60-95^{\circ} \mathrm{C}$ and the temperature was raised by $1^{\circ} \mathrm{C}$ per $20 \mathrm{sec}$. Semilog amplification curves were analyzed using the $2-\Delta \Delta \mathrm{Cq}$ comparative quantification method, and the expression of each gene was normalized to GAPDH (17).

Immunohistochemistry. SK4 and HCN2 overexpression were validated by immunohistochemistry. All the samples for histology were fixed in $4 \%$ paraformaldehyde for $15 \mathrm{~min}$ at room temperature and embedded in paraffin. Sections $(4 \mu \mathrm{m})$ were cut from paraffin blocks of myocardium. The sections were stained with a rabbit anti-HCN2 antibody (1:200; ab84817, Abcam) and a mouse anti-SK4 antibody (1:200; ab219108, Abcam) overnight at $4^{\circ} \mathrm{C}$. The sections were then incubated with Cy3-conjugated goat anti-mouse IgG (1:50, AS-1112, Aspen Technology, Inc.; red fluorescence for SK4) and Alexa Fluor ${ }^{\circledR}$ 488-conjugated goat anti-rabbit IgG (1:50, AS-1109, Aspen Technology, Inc.; green fluorescence for HCN2) secondary antibodies for $50 \mathrm{~min}$ at $37^{\circ} \mathrm{C}$. DAPI was used to visualize the nuclei at room temperature for $3 \mathrm{~min}$. Images were obtained from three random visual fields in three different samples. Each slide was examined under a fluorescence microscope (magnification, x200).

Statistical analysis. The data are expressed as the mean \pm standard deviation. The statistical significance of the differences between two groups was determined using 

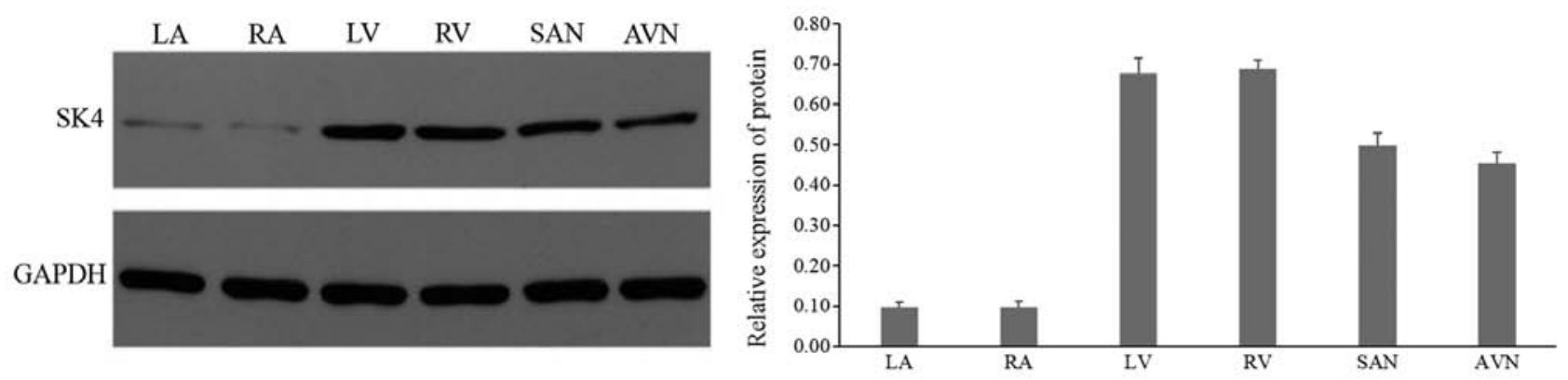

Figure 1. Expression of SK4 in different portions of the rat heart. Western blots of protein sample extracts from the different portion of the normal heart. Antibodies for GAPDH were used as a reference. SK4, intermediate-conductance calcium-activated potassium channel; LA, left atrium; RA, right atrium; LV, left ventricle; RV, right ventricle; SAN, sinoatrial node; $\mathrm{AVN}$, atrioventricular node.

Student's t-test. Comparisons among multiple groups were made using one-way analysis of variance with Turkey tests. $\mathrm{P}<0.05$ was considered to indicate a statistically significant difference. All of the statistical analysis was performed using SPSS 20.0 software (IBM Corp.).

\section{Results}

Expression of SK4 in the rat heart. The expression of SK4 was detected using western blotting in different portions of the Ad-GFP rat heart $(n=10)$; the expression level of SK4 in the ventricle area was the highest, followed by the sinoatrial node and the atrioventricular node area. The expression was lowest in the atrial area (Fig. 1).

Ex vivo complete heart block model and origin of ectopic rhythm. The atrioventricular node region was ablated with $75 \%$ alcohol using a microsyringe. After the atrioventricular node was ablated, the electrocardiogram showed obvious atrioventricular dissociation, and the heart rate was clearly slowed. After the atrioventricular node was ablated, all of the isolated perfused hearts showed junctional escape rhythm or ventricular rhythm (Fig. 2). Compared with the spontaneous electrocardiogram after complete heart block and the pacing electrocardiogram of the transgene site, it was observed that the hearts in the GFP and SK4 groups showed junctional escape rhythms (Fig. 2A and B). Furthermore, the pacing electrocardiogram at the transgene site was markedly different compared with the spontaneous electrocardiogram (Fig. 2A and B). By contrast, the pacing electrocardiograms at the transgene sites in the HCN2 $(n=8)$ and the SK4/HCN2 $(n=7)$ groups were similar to the corresponding spontaneous electrocardiograms, and exhibited a ventricular rhythm (Fig. 2C and D). As opposing $\mathrm{P}$ wave axes were observed in the GFP group, and the SK4 and HCN2 groups, it was hypothesized that the $\mathrm{P}$ waves may change polarity during the process of atrioventricular block, due to the heterogeneity of conduction velocity in the heart. Compared with the GFP $[96.7 \pm 7.6$ beats per min (BPM; maximum, 105 BPM; minimum, 80 BPM)] and SK4 groups [98.1 \pm 8.9 BPM (maximum, 105 BPM; minimum, 74 BPM)], the spontaneous heart rates in the HCN2 group were significantly increased [111.7 \pm 5.5 BPM (maximum, 123 BPM; minimum, 103 BPM); $\mathrm{P}<0.05$ vs. GFP and SK4], and the heart rates in the SK4/HCN2 group were further increased compared with the HCN2 group [139.9 \pm 21.9 BPM (maximum, 217 BPM; minimum, 120 BPM); $\mathrm{P}<0.05$, Table II]. To determine the stability of heart rates after complete heart block was established, heart rate variability with an assessment of the standard deviation of NN intervals (SDNN). The SDNNs of the four groups are presented in Table III. It was determined that the SDNN was not significantly affected by SK4 and/or HCN2 overexpression.

APD alternans and VA inducibility. To evaluate the risk of VA, APD alternans and VA inducibility were measured. The threshold of APD alternans was not statistically significant between the various groups. Additionally, neither non-sustained ventricular tachycardia nor ventricular fibrillation was induced in each group, and the incidence of ventricular premature contraction was not significantly different between the groups (Table IV).

Expression levels of SK4 and HCN2. Myocardial tissue was extracted from the transgene site to detect the expression of SK4 and HCN2 protein, and the corresponding genes HCN2 and KCNN4 (Figs. 3 and 4). Western blotting revealed that the expression levels of SK4 were higher in the SK4 group $(0.83 \pm 0.04)$ and the SK4/HCN2 group $(0.84 \pm 0.04)$ compared with in the GFP group $(0.45 \pm 0.03$; $\mathrm{P}<0.05$; Fig. $3 \mathrm{~A})$; however, there was no significant difference between the SK4 and SK4/HCN2 groups ( $>0.05)$, nor between the HCN2 and GFP groups. Similarly, the expression levels of HCN2 in the HCN2 group $(0.65 \pm 0.03)$ and the SK4/HCN2 group $(0.67 \pm 0.02)$ were higher than in the GFP group $(0.15 \pm 0.01 ; \mathrm{P}<0.05$; Fig. $3 \mathrm{~A})$. There was no significant difference between the HCN2 and the SK4/HCN2 groups (P>0.05), or the SK4 and GFP groups $(\mathrm{P}>0.05)$. The RT-qPCR results demonstrated similar expression profiles at the mRNA level (Fig. 3B). SK4 and HCN2 overexpression were further validated by immunohistochemistry. As presented in Fig. 4, SK4 (red) and HCN2 (green) were both overexpressed in tissue from the SK4, HCN2 and SK4/HCN groups compared with the GFP group.

\section{Discussion}

In the present study, biological pacemaker activity was successfully generated via the overexpression of SK4/HCN2 in a rat model of complete heart block. The results showed the following: i) SK4 and HCN2 were successfully expressed 
A GFP group heart: junctional escape rhythm

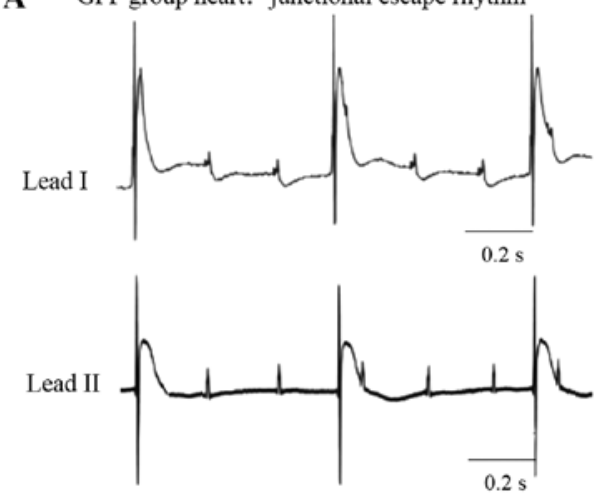

B Sk4 group heart: junctional escape rhythm

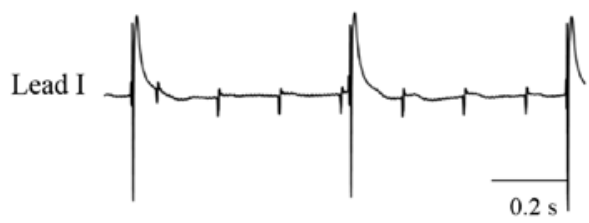

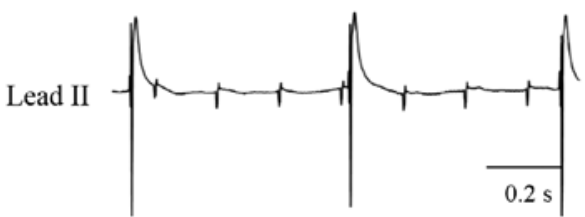

C HCN2 group heart: junctional escape rhythm
Paced at the injection site

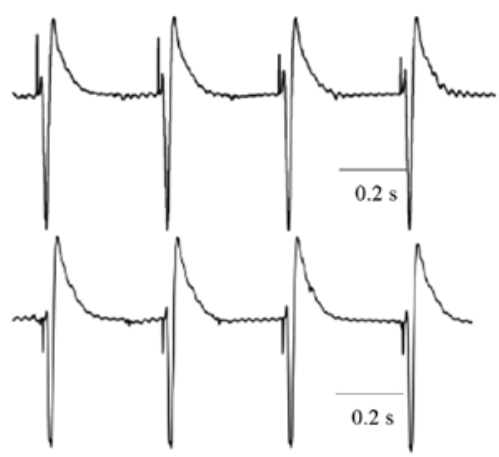

Paced at the injection site
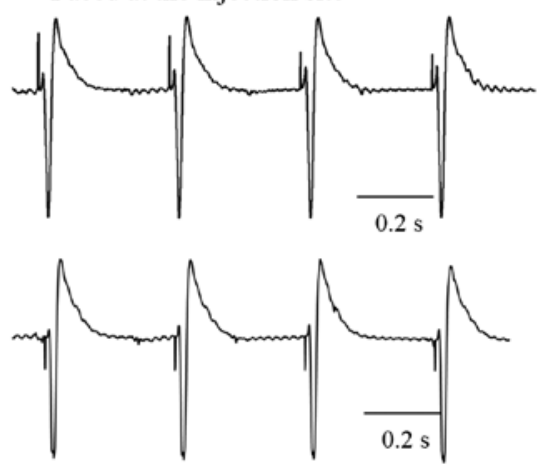

Paced at the injection site

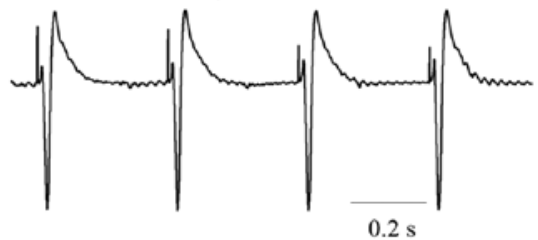

D SK4/HCN2 group heart: junctional escape rhythm

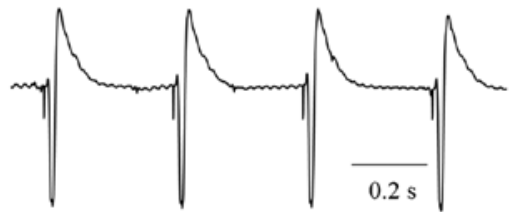

Paced at the injection site

Lead I
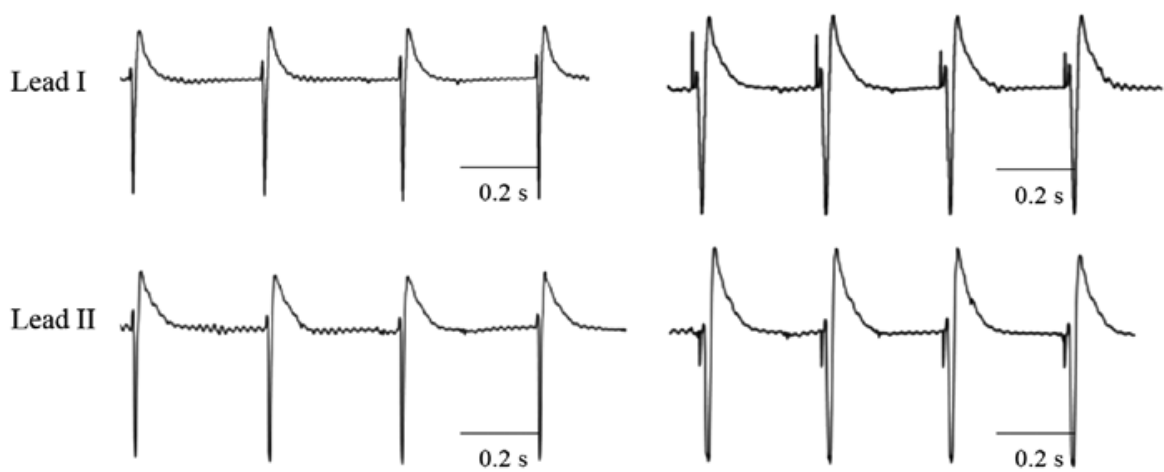

Figure 2. Electrocardiographic recordings of intact perfused hearts. (A) GFP and (B) SK4 group hearts showed a narrow-QRS junctional escape rhythm, the opposite pattern to those of electrode-paced beats at the injection site. The morphology of the ectopic beats in (C) HCN2 and (D) SK4/HCN2 group were very similar to those of the electrode-paced beats at the transgene site. GFP, green fluorescent protein; HCN2, hyperpolarization-activated cyclic nucleotide-gated channel 2; SK4, intermediate-conductance calcium-activated potassium channel.

in the myocardium after the adenoviral vectors was injected; ii) $\mathrm{SK} 4 / \mathrm{HCN} 2$ co-overexpression could generate an ectopic rhythm in the ventricle; and iii) the pacing efficiency in the
SK4/HCN2 co-overexpression group was improved compared with that in the groups with overexpression of HCN2 or SK4 alone. 
Table II. Heart rate after a complete heart block was established between groups.

\begin{tabular}{lcccr}
\hline Groups & GFP & SK4 & HCN2 & SK4/HCN2 \\
\hline Heart rate (beats/minute) & $96.7 \pm 7.6$ & $98.1 \pm 8.9$ & $111.7 \pm 5.5^{\mathrm{a}}$ & $139.9 \pm 21.9^{\mathrm{a}, \mathrm{b}}$ \\
\hline
\end{tabular}

Data analyzed using one-way ANOVA; GFP, green fluorescent protein; SK4, intermediate-conductance calcium-activated potassium channel; $\mathrm{HCN} 2$, hyperpolarization-activated cyclic nucleotide-gated channel 2 ; ${ }^{\mathrm{a}} \mathrm{P}<0.05$ vs. GFP or $\mathrm{SK} 4,{ }^{\mathrm{b}} \mathrm{P}<0.05$ vs. HCN2.

Table III. Heart rate variability after a complete heart block was established between groups.

\begin{tabular}{lccccc}
\hline Groups & GFP & SK4 & HCN2 & SK4/HCN2 & P-value \\
\hline SDNN (mean \pm standard deviation, msec) & $0.52 \pm 0.07$ & $0.50 \pm 0.06$ & $0.55 \pm 0.05$ & $0.53 \pm 0.06$ & P $>0.05$ for all
\end{tabular}

Data analyzed using one-way ANOVA; P>0.05. GFP, green fluorescent protein; HCN2, hyperpolarization-activated cyclic nucleotide-gated channel 2; SK4, intermediate-conductance calcium-activated potassium channel; SDNN, standard deviation of NN intervals.

Table IV. APD alternans and VA inducibility between groups.

\begin{tabular}{lcccrr}
\hline Groups & GFP & SK4 & HCN2 & SK4/HCN2 & P-value \\
\hline APD alternans (msec) & $82.5 \pm 8.9$ & $83.8 \pm 10.6$ & $83.8 \pm 7.4$ & $82.5 \pm 12.8$ & P $>0.05$ for all \\
VA inducibility & $3 / 30$ & $5 / 30$ & $4 / 30$ & $4 / 30$ & P>0.05 for all \\
\hline
\end{tabular}

Data analyzed using one-way ANOVA; P $>0.05$. GFP, green fluorescent protein; HCN2, hyperpolarization-activated cyclic nucleotide-gated channel 2; SK4, intermediate-conductance calcium-activated potassium channel; APD, action potential duration alternans; VA, ventricular arrhythmia.

SK4 previously was found to be expressed in the mouse SAN (6), ESC-CM (4,5), adult human SAN (6) and right atrium, and in ventricular biopsies (2). The present study found that SK4 was expressed in the rat atrium, ventricle, SAN and atrioventricular node, with the highest expression levels observed in the ventricle. A previous study has shown that after the SK4 channel was specifically inhibited, the MDP of cardiomyocytes was depolarized but the duration of APD50 was unaltered, indicating that SK4 currents do not notably affect the action potential repolarization duration and only act on the late stage of repolarization (5).

Previous studies have shown that overexpression or activation of SK4 in embryonic stem cell-derived cardiomyocytes (ESC-CM) can increase the APD, trigger frequency and beating area of the embryoid body $(3,4)$; a mathematical model predicted that the trigger frequency of the sinoatrial node cells could be increased by increasing SK4 current (6). Therefore, the improved biological pacing activity induced by SK $4 / \mathrm{HCN} 2$ co-overexpression in the present study may be regulated by the outward SK4 potassium current. This current is responsible for the notch of the MDP, providing a driving force that is sufficiently strong to activate the I(f) current at the early phase of the DD slope; SK4 synergistically interacts with I(f) by maintaining the voltage changes within a range where $\mathrm{HCN}$ channels can most effectively operate (2). It is proposed that this phenomenon is termed SK4-induced I(f) activation. However, the overexpression of SK4 alone cannot generate biological pacing activity, possibly due to inadequate activation of HCN channels.

Similar to IK1-induced I(f) activation (18), IK1 has long been considered to hyperpolarize cell membranes and inhibit cardiac automaticity; inhibition or knockout of IK1 can increase cardiac automaticity (19). However, it has been observed that co-overexpression of IK1/HCN can significantly increase the beating frequency of left ventricular cardiomyocytes (20) and induce the automaticity of unexcitable 293T cells and ESC-CM cells (21). Electrophysiological data have shown that IK1 can promote an increase in MDP hyperpolarization, accelerate the four-phase depolarization slope and shorten the APD, thus increasing the frequency of I(f)-induced automaticity (18). Other studies indicated that biological pacemaker activity can also be successfully created by overexpression of T-box18 (TBX18) in vivo (22-24). The hypothesized mechanisms underlying this phenomenon are as follows: i) TBX18 can convert ventricular myocytes into sinoatrial node-like cells; and ii) TBX18 drives upregulation of HCN4 or HCN2, and downregulation of connexin 43 , which increases automaticity and the conduction of impulse. The similarities between the above studies and the present study is that $\mathrm{HCN}$ channel is involved; however, unlike TBX18, which affects the expression of $\mathrm{HCN}$, the present study focused on the synergistic effects of SK4 and HCN2 channel overexpression.

Previous studies have shown that the biological heart rates generated by overexpression of $\mathrm{HCN} 212$, Adenylate yclase1 

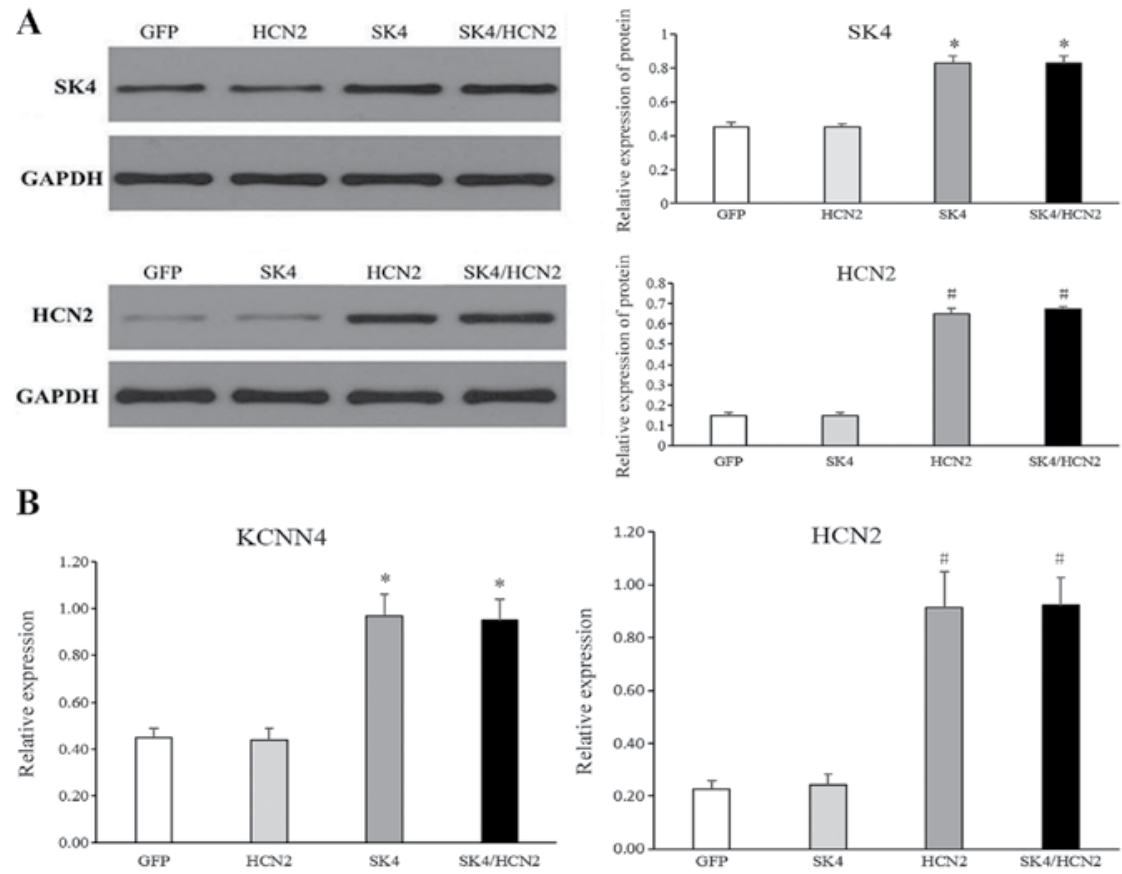

Figure 3. Expression of SK4 and HCN2 in rat hearts following transgene injection. (A) Western blots of protein samples extracted from the ventricular myocardium of the transgene site of the experimental groups. Representative images of western blot results for SK4 (top) and HCN2 (bottom). Antibodies for GAPDH were used as a reference. SK4 (top panel): " $\mathrm{P}<0.05$ vs. GFP or HCN2. HCN2 (bottom panel): "P<0.05 vs. GFP or SK4. (B) Reverse transcription-quantitative $\mathrm{PCR}$ analysis of KCNN4 (left) and $\mathrm{HCN} 2$ (right) in the ventricular myocardium of the transgene site of the experimental groups. KCNN4: ${ }^{*} \mathrm{P}<0.05$ vs. GFP or $\mathrm{HCN} 2 . \mathrm{HCN} 2$ : " $\mathrm{P}<0.05$ vs. GFP or SK4. SK4, intermediate-conductance calcium-activated potassium channel; HCN2, hyperpolarization-activated cyclic nucleotide-gated channel 2; GFP, green fluorescent protein.

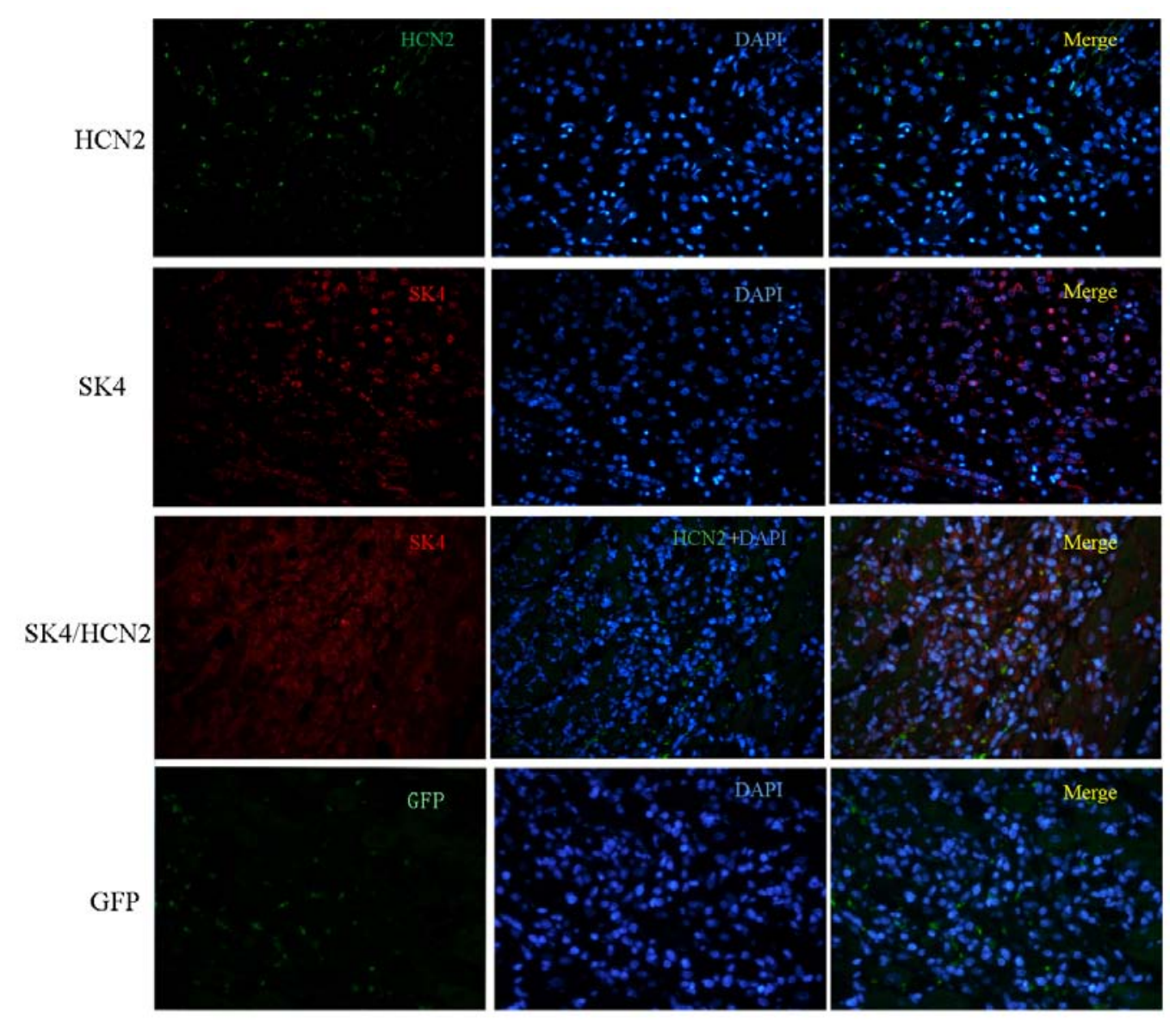

Figure 4. Immunohistochemical staining for SK4 and HCN2. Positive HCN2 (green) and SK4 (red) staining were detected in the injected myocardium from animals that received the corresponding adenovirus (magnification, x200). The nuclei were stained blue using DAPI. HCN2, hyperpolarization-activated cyclic nucleotide-gated channel 2; SK4, intermediate-conductance calcium-activated potassium channel. 
(AC1) and T-box3 far exceed the physiological heart rates and even cause ventricular tachycardia $(9,10,25)$. The present study found that the heart rate of the SK4/HCN2 group increased significantly compared with other groups; however, the fastest heart rate observed was $214 \mathrm{BPM}$, which is remains slower than the normal heart rate of rats (400-500 BPM). This result indicated that the co-overexpression of SK4/HCN2 can safely and successfully generate biological pacing activity. In addition, a study has reported on methods of introducing exogenous genes, such as the overexpression of $\mathrm{AC} 1$ and $\mathrm{HCN} 2 / \mathrm{AC} 1$, that may have proarrhythmic effects, which can occur after depolarization; overexpression can also induce both activity and calcium overload-induced VAs (10). APD alternans is closely associated with VAs, with calcium-triggered calcium release and abnormal intracellular calcium leakage contributing to these effects (26). In the present study, APD alternans and burst pacing-induced VAs were measured, revealed that the threshold of APD alternans between each group was not significantly different. Furthermore, neither non-sustained ventricular tachycardia nor ventricular fibrillation was induced in any group, and the incidence of VA inducibility was not significantly different between the groups (Table II). These results indicated that co-overexpression of SK4/HCN2 does not increase the risk of VAs. However, there are limitations to the present study. First, the myocardium at the transgene site was not isolated, nor were other cellular electrophysiological parameters measured, such as SK4 and I(f) currents, MDP or APD. Data such as these may provide additional information regarding the underlying mechanisms of the observed effects. Additionally, SK4 and HCN2 were not co-expressed at different ratios, as has been performed in other studies (15); such experiments may enable the identification of optimal co-expression ratios for achieving biological pacemaker activity.

In conclusion, biological pacemaker activity can be successfully generated by co-overexpression of SK4 and HCN2 without increasing the risk of VAs. The overexpression of SK4 alone was insufficient to generate biological pacemaker activity. This study provides evidence that SK4 and HCN2 combined could function as an ectopic pacemaker, laying the groundwork for the development of improved biological pacing strategies in the future.

\section{Acknowledgements}

Not applicable.

\section{Funding}

This work was supported by the National Natural Science Foundation of China (grant no. 81670303).

\section{Availability of data and materials}

The datasets used and/or analyzed during the current study are available from the corresponding author on reasonable request.

\section{Authors' contributions}

$\mathrm{HZ}$ and MY conducted the experiments, acquired and analysed the data and drafted the manuscript, with support from QZ and $\mathrm{CH}$. HZ, FW and AY contributed to sample preparation, performed the calculations and designed the figures. XW, YT and TW conceived the study and supervised the project. All authors discussed the results and contributed to the final manuscript.

\section{Ethics approval and consent to participate}

This study was approved by the Animal Studies Subcommittee of Wuhan University School of Medicine and conducted in accordance with the Guide for the Care and Use of Laboratory Animals of the National Institutes of Health.

\section{Patient consent for publication}

Not applicable.

\section{Competing interests}

The authors declare that they have no competing interests.

\section{References}

1. Vergara C, Latorre R, Marrion NV and Adelman JP: Calcium-activated potassium channels. Curr Opin Neurobiol 8: 321-329, 1998.

2. Weisbrod D, Khun SH, Bueno H, Peretz A and Attali B: Mechanisms underlying the cardiac pacemaker: The role of SK4 calcium-activated potassium channels. Acta Pharmacol Sin 37: 82-97, 2016.

3. Kleger A, Seufferlein T, Malan D, Tischendorf M, Storch A, Wolheim A, Latz S, Protze S, Porzner M, Proepper C, et al: Modulation of calcium-activated potassium channels induces cardiogenesis of pluripotent stem cells and enrichment of pacemaker-like cells. Circulation 122: 1823-1836, 2010.

4. Liebau S, Tischendorf M, Ansorge D, Linta L, Stockmann M, Weidgang C, Iacovino M, Boeckers T, von Wichert G, Kyba M and Kleger A: An inducible expression system of the calcium-activated potassium channel 4 to study the differential impact on embryonic stem cells. Stem Cells International 2: 456815, 2011.

5. Weisbrod D, Peretz A, Ziskind A, Menaker N, Oz S, Barad L, Eliyahu S, Itskovitz-Eldor J, Dascal N, Khananshvili D, et al: SK4 $\mathrm{Ca}^{2+}$ activated $\mathrm{K}+$ channel is a critical player in cardiac pacemaker derived from human embryonic stem cells. Proc Natl Acad Sci USA 110: E1685-E1694, 2013.

6. Haron-Khun S, Weisbrod D, Bueno H, Yadin D, Behar J, Peretz A, Binah O, Hochhauser E, Eldar M, Yaniv Y, et al: SK4 $\mathrm{K}+$ channels are therapeutic targets for the treatment of cardiac arrhythmias. EMBO Mol Med 9: 415-429, 2017.

7. Lai MH, Wu Y, Gao Z, Anderson ME, Dalziel JE and Meredith AL: SK4 $\mathrm{Ca}^{2+}$ activated $\mathrm{K}+$ channels regulate sinoatrial node firing rate and cardiac pacing in vivo. Bio J 112: 35a, 2017.

8. Bucchi A, Plotnikov AN, Shlapakova I, Danilo P Jr, Kryukova Y, Qu J, Lu Z, Liu H, Pan Z, Potapova I, et al: Wild-type and mutant HCN channels in a tandem biological-electronic cardiac pacemaker. Circulation 114: 992-999, 2006.

9. Plotnikov AN, Bucchi A, Shlapakova I, Danilo P Jr, Brink PR, Robinson RB, Cohen IS and Rosen MR: HCN212-channel biological pacemakers manifesting ventricular tachyarrhythmias are responsive to treatment with I(f) blockade. Heart Rhythm 5: 282-288, 2008

10. Boink GJ, Nearing BD, Shlapakova IN, Duan L, Kryukova Y, Bobkov Y, Tan HL, Cohen IS, Danilo P Jr, Robinson RB, et al: $\mathrm{Ca}(2+)$-stimulated adenylyl cyclase AC1generates efficient biological pacing as single gene therapy and in combination with HCN2. Circulation 126: 528-536, 2012.

11. Miake J, Marbán E and Nuss HB: Biological pacemaker created by gene transfer. Nature 419: 132-133, 2002.

12. Cingolani E, Yee K, Shehata M, Chugh SS, Marbán E and Cho HC: Biological pacemaker created by percutaneous gene delivery via venous catheters in a porcine model of complete heart block. Heart Rhythm 9: 1310-1318, 2012.

13. Boink GJ, Duan L, Nearing BD, Shlapakova IN, Sosunov EA, Anyukhovsky EP, Bobkov E, Kryukova Y, Ozgen N, Danilo P $\mathrm{Jr}$, et al: HCN2/SkM1 gene transfer into canine left bundle branch induces stable, autonomically responsive biological pacing at physiological heart rates. J Am Coll Cardiol 61: 1192-1201, 2013. 
14. National Research Council (US) Committee for the Update of the Guide for the Care and Use of Laboratory Animals: Guide for the care and use of laboratory animals, 8th edition. National Academies Press (US), 2011.

15. Qu J, Barbuti A, Protas L, Santoro B, Cohen IS and Robinson RB HCN2 overexpression in newborn and adult ventricular myocytes: Distinct effects on gating and excitability. Circ Res 89: E8-E14, 2001.

16. Baldzizhar A, Manuylova E, Marchenko R, Kryvalap Y and Mary MG: Ventricular tachycardias: Characteristics and management. Crit Care Nurs Clin North Am 28: 317-329, 2016.

17. Livak KJ and Schmittgen TD: Analysis of relative gene expression data using real-time quantitative PCR and the 2(-Delta Delta $\mathrm{C}(\mathrm{T}))$ method. Methods 25: 402-408, 2001.

18. Sun Y, Timofeyev V, Dennis A, Bektik E, Wan X, Laurita KR, Deschênes I, Li RA and Fu JD: A singular role of $I_{K 1}$, promoting the development of cardiac automaticity during cardiomyocyte differentiation by $\mathrm{I}_{\mathrm{k} 1}$-induced activation of pacemaker current. Stem Cell Rev 13: 631-643, 2017.

19. Zaritsky JJ, Redell JB, Tempel BL and Schwarz TL: The consequences of disrupting cardiac inwardly rectifying $\mathrm{K}(+)$ current (I(K1)) as revealed by the targeted deletion of the murine Kir2.1 and Kir2.2 genes. J Physiol 533: 697-710, 2001.

20. Chan YC, Siu CW, Lau YM, Lau CP, Li RA and Tse HF: Synergistic effects of inward rectifier (IK1) and pacemaker (If) currents on the induction of bioengineered cardiac automaticity. J Cardiovasc Electrophysiol 20: 1048-1054, 2010.

21. Chen K, Zuo D, Wang SY and Chen H: Kir2 inward rectification-controlled precise and dynamic balances between Kir2 and HCN currents initiate pacemaking activity. FASEB J 32 3047-3057, 2018.
22. Hu YF, Dawkins JF, Cho HC, Marbán E and Cingolani E: Biological pacemaker created by minimally invasive somatic reprogramming in pigs with complete heart block. Sci Transl Med 6: 245ra94, 2014.

23. Choudhury M, Black N, Alghamdi A, D'Souza A, Wang R, Yanni J, Dobrzynski H, Kingston PA, Zhang H, Boyett MR and Morris GM: TBX18 overexpression enhances pacemaker function in a rat subsidiary atrial pacemaker model of sick sinus syndrome. J Physiol 596: 6141-6155, 2018.

24. Gorabi AM, Hajighasemi S, Khori V, Soleimani M, Rajaei M, Rabbani S, Atashi A, Ghiaseddin A, Saeid AK, Ahmadi Tafti H and Sahebkar A: Functional biological pacemaker generation by T-Box 18 protein expression via stem cell and viral delivery approaches in a murine model of complete heart block. Pharmacol Res 141: 443-450.2019.

25. Hoogaars WM, Engel A, Brons JF, Verkerk AO, de Lange FJ, Wong LY, Bakker ML, Clout DE, Wakker V, Barnett P, et al: Tbx3 controls the sinoatrial node gene program and imposes pacemaker function on the atria. Genes Dev 21: 1098-1112, 2007.

26. Orini M, Hanson B, Taggart $P$ and Lambiase P: Detection of transient, regional carrdiac repolarization alternans by time-frequency analysis of synthetic eletrograms. Eng Med Biol Soc 2013: 3773-3776, 2013.

(i) (9) This work is licensed under a Creative Commons Attribution-NonCommercial-NoDerivatives 4.0 International (CC BY-NC-ND 4.0) License. 